\title{
Minipiloting Tools for Pharmaceutical Development
}

\author{
Julia ZH Gao ${ }^{1} \cdot$ Ajit S. Narang ${ }^{2} \cdot$ Sailesh A. Varia ${ }^{1}$
}

Published online: 5 September 2016

(C) Springer Science+Business Media New York 2016

Minipiloting is an evolving concept that incorporates all aspects of predictable scaling and/or tools used during pharmaceutical development. New chemical entities (NCEs) are tested in pre-clinical stages with de minimus formulation. During early stages of clinical development, a drug in a bottle/capsule formulation approach is commonly used. As development progresses, a more sophisticated dosage form e.g., tablet for administration to patients is needed that would pave the way to a robust larger scale manufacturing. A key objective of pharmaceutical development is to define an acceptable range of incoming material attributes (MA) and unit operation process parameters (PPs) that may impact in-process and finished product quality attributes (QA). These studies are often carried out at a scale of operation that is smaller than pilot and/or proposed commercial scale. The agency defines the pilot scale as 100,000 dosage units or 1/10th of the proposed commercial manufacturing scale - whichever is greater. During early formulation development, the scale of operation often is one or more orders of magnitude smaller than the pilot scale. Utilization of such small scale experiments, characterization tools/techniques, and/or process modeling and simulation to establish process parameters and/or predict performance of inprocess material, and finished product attributes at the large scale is defined herein as the minipiloting. The scale of operation at which such information/data is generated is defined as the minipilot scale.

Minipiloting tools can include, for example, the following:

Julia ZH Gao

zhihui.gao@bms.com

1 Drug Product Science and Technology, Bristol Myers Squibb Company, New Brunswick, NJ, USA

2 Genentech, Inc., San Francisco, CA, USA a) Investigative techniques that can be useful for identification of potential risks as drug product (DP) manufacturing process are scaled-up. These include benchtop-scale studies to gain product and process knowledge that help predict the behavior (scale-up), assess risks (Risk Analysis), and diagnose production issues.

b) Predictive models that can help quantify desired input MAs and PPs that result in desirable in-process MAs and DS (drug substance)/DP QAs.

This issue of JPI contains articles that highlight these two types of minipiloting tools.

Pingali et al. at the Western Michigan University discuss the utility of a minipiloting tool to assess powder flow and charge accumulation tendency as a function of shear stress at a small scale.

Zettler et al. describe the predictive ability of laboratoryscale powder flow characterization on process performance at manufacturing scale. Authors identified how different types of consolidation stresses at the manufacturing scale limited the predictive ability of flow characterization tools. They also presented trends and correlations that could further guide the formulation scientists in understanding and establishing predictive minipiloting tools for powder flow.

Chen et al. focus on the use of mechanistic models in the development of ready-to-use solution and lyophilized products for parenteral use. These models are built on first principles using techniques such as CFD, FEM etc., to address complexities enabling greater process understanding across scales of operation.

Swaminathan et al. describe a framework for measuring the powder Drucker-Prager Cap (DPC) parameters and using them in a finite element method (FEM) simulation for investigating the formation of a debossed surface features on tablet. It is the first step in developing a fully predictive model for 
investigating the design of debossed features to reduce common problems associated with debossing such as picking and illegible features.

Ramachandran et al. describe a multidimensional compartmental population balance model (PBM) coupled with discrete element method simulations to evaluate mecha- nistic rate expressions describing aggregation, breakage, consolidation, and particle flow for a twin screw granulation process. The model's qualitative ability to predict the effects of screw element design and configuration on granule size distribution, porosity, and liquid distribution is highlighted in this paper. 